\title{
Sevoflurane anesthesia in pregnant rats negatively affects nerve function in offspring potentially via inhibition of the $\mathbf{W n t} / \boldsymbol{\beta}$-catenin pathway
}

\author{
YIYAO WANG, YU LI, QUNZHI XING, XUECHAN G. HAN, XU DONG, YIPING LU and MINTAO ZHOU \\ Department of Anesthesiology, The First Affiliated Hospital and College of Clinical Medicine, \\ Henan University of Science and Technology, Luoyang, Henan 471003, P.R. China
}

Received December 11, 2015; Accepted January 10, 2017

DOI: $10.3892 / \mathrm{mmr} .2017 .6316$

\begin{abstract}
Due to the rapid development of medical technology used to perform intrauterine procedures during pregnancy, the number of patients receiving fetal surgery under general anesthesia is increasing. The aim of the present study was to determine the effects of anesthetics on the offspring of rats, and to identify the potential mechanisms underlying these effects. On day 14 of pregnancy, Sprague-Dawley rats were equally divided into the following 3 groups $(n=9)$ : Control group $(n=3), 3 \%$ sevoflurane group $(n=3)$ and $4 \%$ sevoflurane group $(n=3)$. Following birth of the offspring, the juvenile rats were assessed using an open-field test, Morris water maze and a continuous passive avoidance test on different days to determine their learning abilities and memory. Western blot and reverse transcription-quantitative polymerase chain reaction (RT-qPCR) analyses were used to examine the expression of multiple critical factors associated with the proliferation and apoptosis of nerve cells, including Ki67, nestin, B cell leukemia/lymphoma 2 (Bcl-2), BCL2 associated X (Bax) and caspase-3. Additionally, the level of adenosine triphosphate production among the 3 groups were compared. Furthermore, expression alterations in of glycogen synthase kinase-3 $\beta$ (GSK-3 $\beta$ ) and $\beta$-catenin were examined. The Morris water maze experiment revealed that an increased concentration of sevoflurane exposure significantly reduced the learning and memory abilities of the juvenile rats when compared with controls. In addition, western blotting and RT-qPCR analyses determined that the protein and mRNA expression levels of Bax, caspase- 3 and GSK-3 $\beta$ were significantly increased relative to the controls. By contrast, the expression levels of nestin,
\end{abstract}

Correspondence to: Professor Qunzhi Xing, Department of Anesthesiology, The First Affiliated Hospital and College of Clinical Medicine, Henan University of Science and Technology, 24 Jinghua Road, Jianxi, Luoyang, Henan 471003, P.R. China E-mail: xingqunzhi123@tom.com

Key words: sevoflurane, juvenile rats, learning, memory, Wnt $/ \beta$-catenin signaling pathway
$\mathrm{Ki}-67, \mathrm{Bcl}-2$ and $\beta$-catenin were significantly reduced. The results of the present study suggest that exposure of pregnant mice to sevoflurane anesthesia demonstrates a negative effect on the learning and memory abilities of their offspring, and the Wnt/ $\beta$-catenin signaling pathway may be involved in this process.

\section{Introduction}

The number of patients undergoing surgery during pregnancy is increasing, and an increasing number of studies are investigating the effects of anesthesia during pregnancy $(1,2)$. Anesthetic exposure, particularly during the critical stages of neurodevelopment, is considered to be safe and without any adverse long-term consequences (3). However, previous animal studies suggest that exposure to certain anesthetics during periods of rapid synaptogenesis may evoke neuronal apoptosis and inhibit neurogenesis, which may lead to lasting cognitive impairments in the offspring (4). Therefore, it is necessary to investigate the effects and underlying mechanisms of anesthetic exposure on the offspring of rats that have undergone anesthesia during pregnancy.

Sevoflurane is a volatile anesthetic and demonstrates numerous advantages over other intravenous or inhalation anesthetics, including reduced discomfort and a lower blood solubility that facilitates rapid induction and recovery (5). Additionally, it is broadly used in a number of clinical applications (6-8). Previous studies have been performed to determine the effects of sevoflurane on brain development in rodents; the majority of these studies have determined that sevoflurane exposure led to increased apoptosis of brain cells $(9,10)$. However, the precise effect of sevoflurane on the offspring remains inconclusive, and the underlying mechanisms remain to be elucidated.

The Wnt/ $\beta$-catenin signaling pathway is a crucial regulator of the survival, growth and differentiation of neurons (11). A previous study reported that activation of the Wnt/ $\beta$-catenin pathway improves cell replacement therapy for Parkinson's disease (12). In addition, Wnt/ $\beta$-catenin signaling regulates hippocampal neurogenesis, with Wnt-knockout mice exhibiting midbrain neuronal loss (13). Furthermore, the Wnt/ $\beta$-catenin pathway regulates oligodendrocyte 
development in a stage-dependent manner (14). Glycogen synthase kinase-3 $\beta$ (GSK-3 $\beta$ ) is a central downstream factor of the Wnt/ $\beta$-catenin pathway. A previous study demonstrated that GSK-3 $\beta$-mediated inhibition of $\beta$-catenin potentially leads to astrogliosis and inflammation resulting in neural stem cell survival (15).

The molecular mechanisms involved in neuronal growth and differentiation remain to be fully elucidated. The present study investigated the effects of sevoflurane on the neurological functions of the offspring of rats exposed to sevoflurane during pregnancy, as well as the molecular mechanisms underlying these effects.

\section{Materials and methods}

Animal model. The study protocol was approved by the Medical Ethics and Human Clinical Trial Committee of Henan University of Science and Technology (Luoyang, China). The procedures were approved by the institutional Animal Research Ethics Committee of Henan University of Science and Technology with reference to the Chinese Community guidelines for the use of experimental animals (16). On day 14 of pregnancy, a total of 27 female Sprague-Dawley rats (6-8 weeks old; average weight $200 \mathrm{~g}$ ) that were obtained from the Laboratory Animal Centre of Henan University of Science and Technology, and raised at room temperature with freely available food and water and $12 \mathrm{~h}$ day/night cycle, were divided into 3 groups (n=9): Control group (group C), 3\% sevoflurane group and $4 \%$ sevoflurane group using the random digit table method. The 3 groups were exposed to air, $3 \%$ sevoflurane and $4 \%$ sevoflurane (Sangon Biotech Co., Ltd., Shanghai, China) for $4 \mathrm{~h}$, respectively. At 35 days of age, the juvenile rats were examined using an open-field test and Morris water maze experiments as previously reported (17). The time taken to cross the original platform area within $90 \mathrm{sec}$ was automatically recorded by image acquisition and analysis systems (XR-XM101; Shanghai Xinruan Information Technology Co., Ltd., Shanghai, China) as previously reported (17). At 42 days of age, juvenile rats were examined using the continuous passive avoidance test to determine learning and memory abilities (18). Black boxes were used to measure the fear memory of rats, including training phase as the rats were stimulated by electricity and sound, and the detection phase of memory latency, including context, altered context, pre-conditioning-stimulus and conditioning stimulus tests in Fig. 1. Fear memory was evaluated based on the duration of time that rats spent frozen following stimuli.

Reverse transcription-quantitative polymerase chain reaction $(R T-q P C R)$. Total RNA was isolated from the hippocampus and cerebral cortex of 6-week-old rats using TRIzol reagent (Invitrogen; Thermo Fisher Scientific, Inc., Waltham, MA, USA) following the manufacturer's protocol. cDNA was synthesized by reverse transcribing a total of $20 \mu \mathrm{g}$ RNA using the PrimeScript ${ }^{\mathrm{TM}}$ One Step RT-PCR kit (Takara Biotechnology Co., Ltd., Dalian, China). qPCR was performed using an Mx3000P Real-Time PCR system (Thermo Fisher Scientific, Inc.) according to the manufacturer's protocol, and SYBR Premix Ex Taq (Takara Biotechnology Co., Ltd.) was used as a DNA-specific fluorescent dye. The primers used for the detection of target mRNA expression were synthesized in our lab, and the sequences are presented in Table I. Reactions were repeated $>3$ times. Gene expression levels were calculated by $2^{-\triangle \Delta C q}$ method (19) relative to $\beta$-actin using Stratagene Mx3000P software (Agilent Technologies, Inc., Santa Clara, CA, USA).

Western blotting. In order to determine protein expression levels, whole cell extracts (lysate) were prepared from rat brain tissues (6-weeks-old) in RIPA lysis buffer [50 mM Tris- $\mathrm{HCl}$ (pH 7.4), $150 \mathrm{mM} \mathrm{NaCl}, 1 \% \mathrm{NP}-40$ and $0.1 \%$ SDS]. The concentration of protein was determined by the BCA method. Total protein $(30 \mu \mathrm{g})$ from each sample was separated on $12 \%$ SDS-PAGE gels and transferred onto a nitrocellulose membrane. Target proteins were probed with specific primary antibodies for nestin (1:1,000; cat. no. ab18102; Abcam, Cambridge, UK), Ki67 (1:1,000; cat. no. ab92353; Abcam), GSK-3 $\beta$ (1:1,000; cat. no. 12456; Cell Signaling Technology, Inc., Danvers, MA, USA) $\beta$-catenin (1:1,000; cat. no. 9582; Cell Signaling Technology, Inc.), caspase 3 (1:1,000; cat. no. SC-271759, Santa Cruz Biotechnology, Inc., Dallas, TX, USA), BCL2 associated X (Bax; 1:200; cat. no. sc-4239; Santa Cruz Biotechnology, Inc., Dallas, TX, USA), B cell leukemia/lymphoma 2 (Bcl-2; 1:200; cat. no. sc-7382; Santa Cruz Biotechnology, Inc.) and $\beta$-actin (1:500; cat. no. sc-1615; Santa Cruz Biotechnology, Inc.) at $4^{\circ} \mathrm{C}$ overnight. Subsequently, the membrane was incubated with Goat Anti-Mouse IgG (H\&L) secondary antibody (1:5,000; cat. no. PAB0096; Abnova, Taipei, Taiwan) or Anti-Rabbit IgG (H+L) secondary antibody (1:5,000; cat. no. 14708; Cell Signaling Technology, Inc.) at room temperature for $1 \mathrm{~h}$. Non-specific binding sites were blocked by immersing the membrane into $5 \%$ non-fat milk in $0.1 \%$ Tween 20-TBS (TTBS) solution for $1 \mathrm{~h}$ at room temperature with shaking. The membrane was visualized by an ECL kit (Beyotime Institute of Biotechnology) on the GelDoc $^{\mathrm{TM}}$ XR+ system (Bio-rad Laboratories, Inc., Hercules, CA, USA).

Adenosine triphosphate (ATP) production assay. ATP production in 3-week-old rat brain tissues was analyzed using an ATP assay kit (Beyotime Institute of Biotechnology) according to the manufacturer's instructions.

Immunofluorescence staining. Rats were sacrificed and the brain cortex was extracted. After fixing in $10 \%$ formaldehyde for $1 \mathrm{~h}$ at room temperature, pathological slides $(5 \mu \mathrm{m}$-thick sections) were deparaffinized in xylene and rehydrated in decreasing concentrations of ethanol to water. Then all the reactions were performed as described previously (20). Sections were immunostained with primary antibody against Ki67 (1:100; cat. no. ab92353; Abcam) and horseradish peroxidase-conjugated Goat Anti-Mouse IgG secondary antibody (1:50; cat. no. sc-2005; Santa Cruz Biotechnology, Inc.).

Statistical analysis. Data were analyzed using SPSS software (version, 17.0; SPSS, Inc., Chicago, IL, USA). One-way analysis of variance was used to compare differences among treatment groups. Experiments were performed independently at least three times. $\mathrm{P}<0.05$ was considered to indicate a statistically significant difference. 
Table I. Primer sequences used for reverse transcription-quantitative polymerase chain reaction analysis.

\begin{tabular}{lll}
\hline Gene & \multicolumn{1}{c}{ Forward (5'-3') } & Reverse $\left(5^{\prime}-3^{\prime}\right)$ \\
\hline Nestin & GTAGCTCCCAGAGAGGGG & CTCTAGAGGGCCAGGGAC \\
Ki67 & GCCAAGGGTAACTCGTGG & CCTCCATTGGCGTCTGAAG \\
GSK-3 $\beta$ & GGTGAATCGAGAAGAGCC & CTGTGGTTACCTTGCTGCC \\
$\beta$-catenin & CCATCGAGAGGGCTTGTTG & CAGTACGGAATCCACTGG \\
caspase-3 & GTGAGGCGGTTGTAGAAG & GCTGCATCGACATCTGTACC \\
Bcl-2 & GGTGAACTGGGGGAGGATTG & GGCAGGCATGTTGACTTCAC \\
Bax & GCTGAGCGAGTGTCTCAAG & GTCCAATGTCCAGCCCATG \\
\hline
\end{tabular}

GSK-3 $\beta$, glycogen synthase kinase-3 $\beta$; Bcl2, B-cell CLL/lymphoma; Bax, Bcl2 associated X.
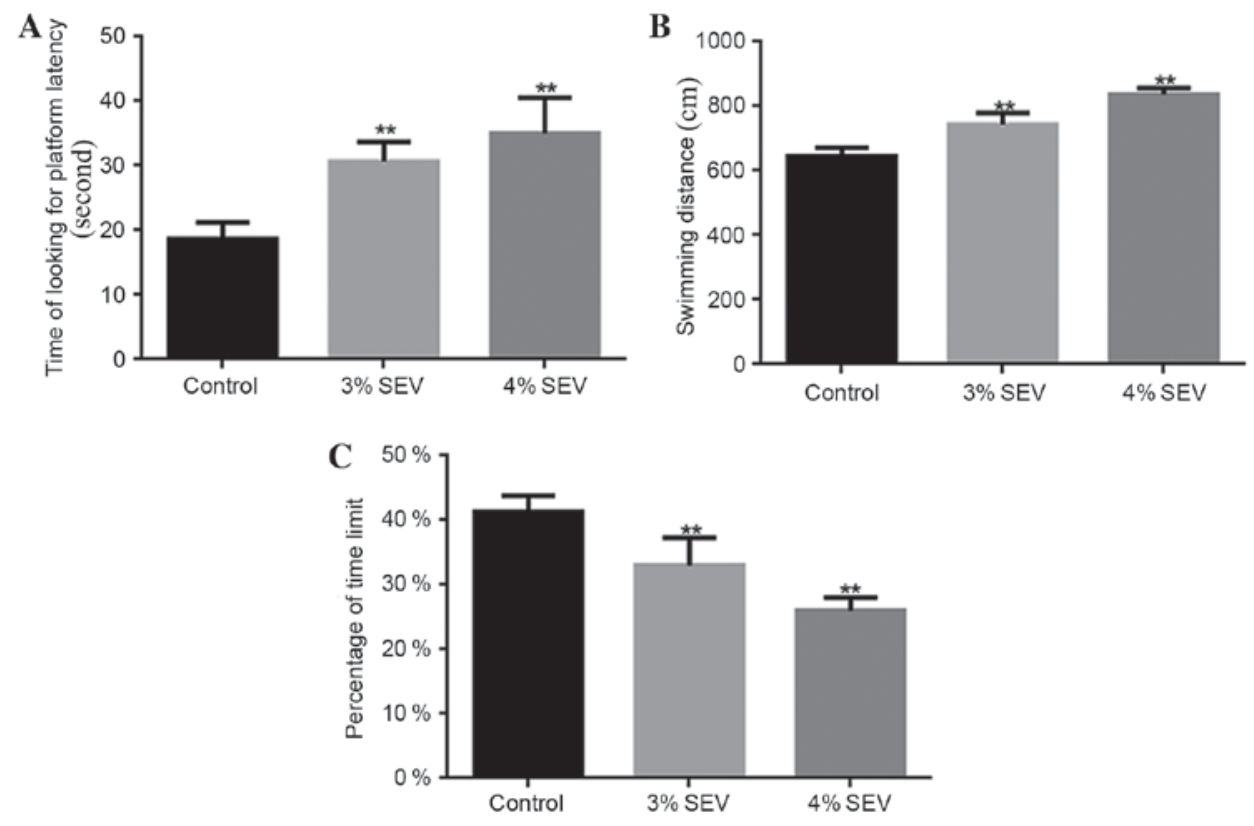

Figure 1. Effects of SEV on learning and memory abilities of juvenile rats. (A) Time taken to locate the platform. (B) Swimming distance. (C) Platform quadrant retention time. ${ }^{* * *} \mathrm{P}<0.05$ vs. control. SEV, sevoflurane.

\section{Results}

Sevoflurane reduces the learning ability and memory in rat offspring. The rat offspring rats that were exposed to 3 and $4 \%$ sevoflurane required a significantly greater amount of time to find the platform when compared with the control group $(\mathrm{P}<0.05$; Fig. 1A). Additionally, the swimming distance during the training period for the offspring of rats exposed to 3 and $4 \%$ sevoflurane was significantly increased when compared with the control group $(\mathrm{P}<0.05$; Fig. 1B). Furthermore, the percentage of platform quadrant retention time was significantly reduced in the offspring of rats exposed to 3 and $4 \%$ sevoflurane when compared with the control group $(\mathrm{P}<0.05$; Fig. 1C). The results demonstrated that sevoflurane reduced the learning and memory abilities of the offspring of rats exposed to sevoflurane during pregnancy.

Sevoflurane inhibits the proliferation of nerve cells in the offspring rats. The protein and mRNA expression levels of nestin and Ki67 were significantly reduced in groups $\mathrm{S} 1$ and $\mathrm{S} 2$ compared with group C (Fig. 2A and B; P<0.05). Additionally, the expression level of $\mathrm{Ki}-67$ was reduced in the cerebral cortex of the offspring rats in the 3 and $4 \%$ sevoflurane groups in contrast with the group $\mathrm{C}$ (Fig. 2C). These findings demonstrated that sevoflurane inhibited the proliferation of nerve cells in the offspring of rats exposed during pregnancy.

Sevoflurane promotes nerve cell apoptosis in rat offspring. ATP production was significantly reduced in the offspring of rats exposed to 3 and $4 \%$ sevoflurane when compared with the control group $(\mathrm{P}<0.05$; Fig. $3 \mathrm{~A})$. Additionally, the mRNA and protein expression levels of Bax and caspase- 3 were significantly increased in the neuronal cells of the offspring of rats exposed to 3 and $4 \%$ sevoflurane when compared with the control group $(\mathrm{P}<0.05$; Fig. $3 \mathrm{~B}$ and $\mathrm{C})$. By contrast, the expression level of Bcl-2 was significantly reduced in sevoflurane-treated groups when compared with the control group $(\mathrm{P}<0.05$; Fig. 3B). These findings suggest that sevoflurane may 

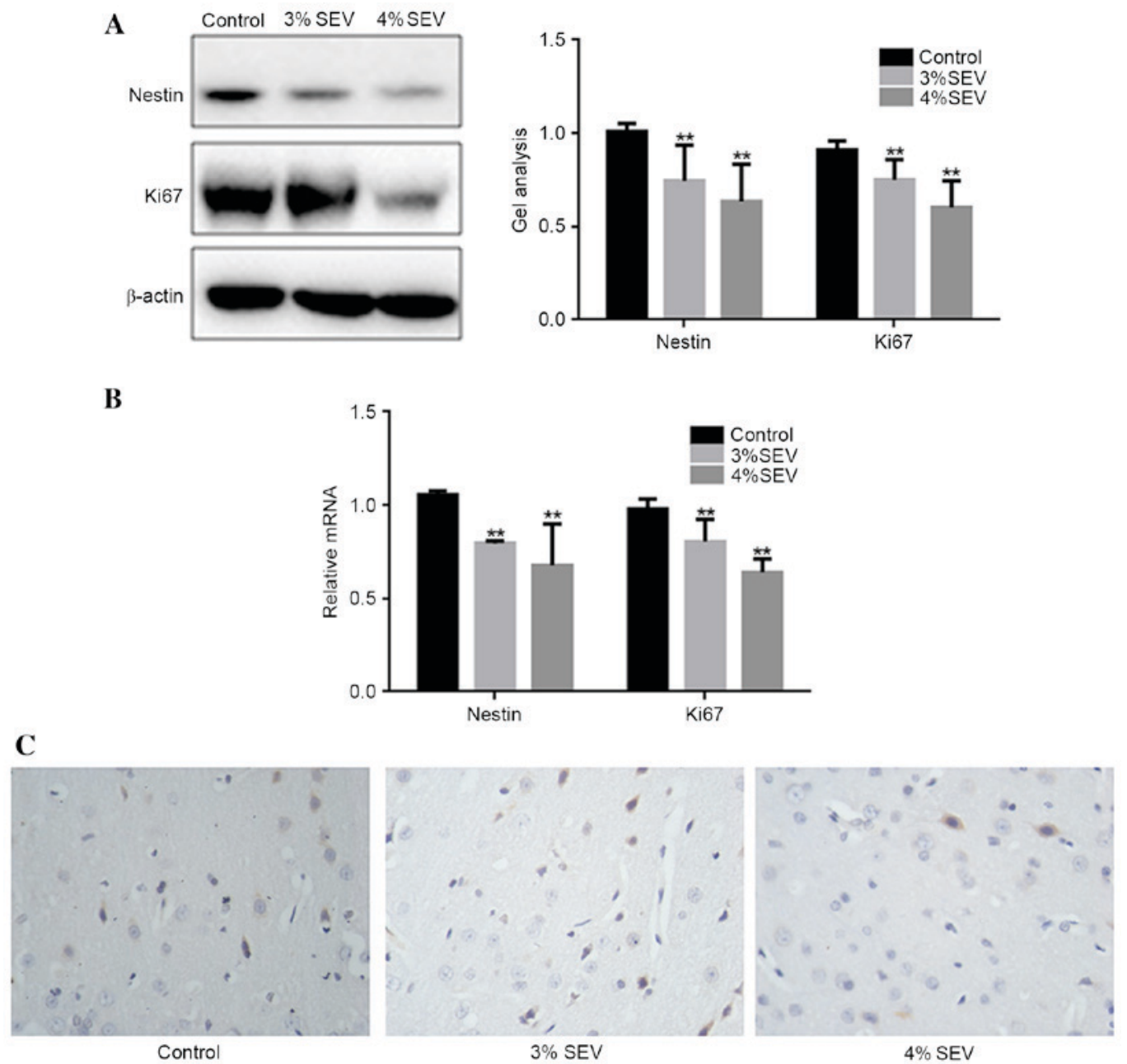

Figure 2. Effects of SEV on the proliferation of the neurons in juvenile rats. The (A) protein and (B) mRNA expression levels of nestin and Ki67 as determined by western blotting and reverse transcription-quantitative polymerase chain reaction analyses, respectively. (C) Expression of Ki67 in the cerebral cortex of neonatal rats. $\mathrm{x} 400$, magnification. ${ }^{* *} \mathrm{P}<0.05$ vs. control. SEV, sevoflurane.

promote nerve cell apoptosis in the offspring of rats exposed during pregnancy.

Sevoflurane inhibits the growth of nerve cells in rat offspring potentially via the Wnt/ $\beta$-catenin signaling pathway. The protein and mRNA expression levels of GSK- $3 \beta$ and $\beta$-catenin were significantly increased in the offspring of rats exposed to 3 and $4 \%$ sevoflurane when compared with the control group ( $\mathrm{P}<0.05$; Fig. 4). These results suggest that sevoflurane may inhibit the growth of nerve cells in the offspring of exposed rats via the Wnt/ $/$-catenin signaling pathway.

\section{Discussion}

Sevoflurane is one of the most widely used inhalation anesthetics for pediatric surgery and cesarean delivery due to rapid inhalational induction, tolerable odor, quick emergence and relative cardiovascular stability for infants and children (21). Although sevoflurane exhibits fewer severe effects on the developing brains of animals compared with isoflurane, which is a structurally similar inhaled anesthetic (22), previous studies have demonstrated that sevoflurane may lead to neuronal apoptosis and behavioral dysfunction $(23,24)$.
The present study assessed the neurobehavioral effects of sevoflurane on the offspring of exposed pregnant rats using the open-field test and the Morris water maze. The results demonstrated the learning and memory abilities of the offspring rats were significantly reduced in sevoflurane-exposed groups. In addition, the western blotting and RT-qPCR analysis results revealed that the protein and mRNA expression levels of Bax and caspase-3 were significantly increased in sevoflurane-exposed groups when compared with the controls. These results suggest that sevoflurane promoted nerve cell apoptosis in rat offspring, which is consistent with the results of previous studies $(23,24)$.

The current study determined that the protein and mRNA expression levels of nestin and Ki-67 were significantly reduced in the sevoflurane-treated groups when compared with the controls. Nestin and Ki-67 are important molecules for cell growth, which serves a role in cell division and proliferation (25). It is therefore possible that sevoflurane inhibited the proliferation of nerve cells in the offspring of exposed rats in the present study.

The Wnt/ $\beta$-catenin signaling pathway is a crucial regulator of the growth and proliferation of nerve cells and is widely involved in neuronal development, differentiation, migration, adhesion, polarization and tumorigenesis (26-28). In 

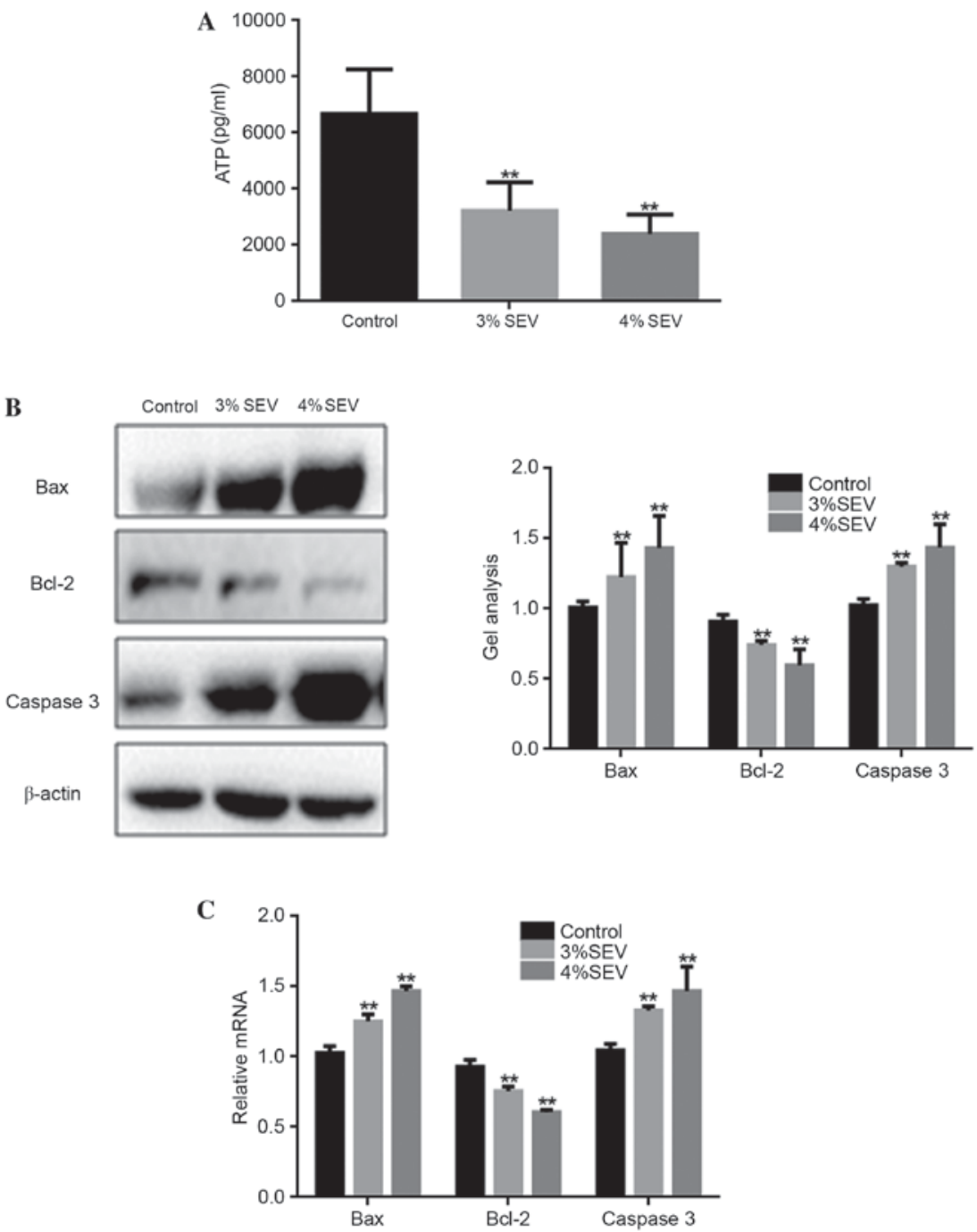

Figure 3. Effects of SEV on neuron apoptosis in juvenile rats. (A) ATP production levels in neurons. The (B) protein and (C) mRNA expression levels of Bax, Bcl-2 and caspase-3. ${ }^{* *} \mathrm{P}<0.05$ vs. control. SEV, sevoflurane; ATP, adenosine triphosphate; Bcl-2, B-cell CLL/lymphoma; Bax, Bcl2 associated X.
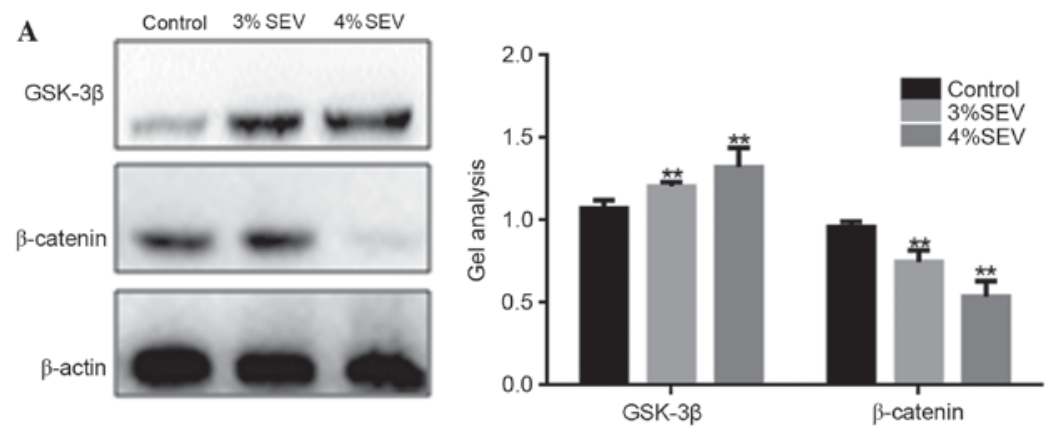

B

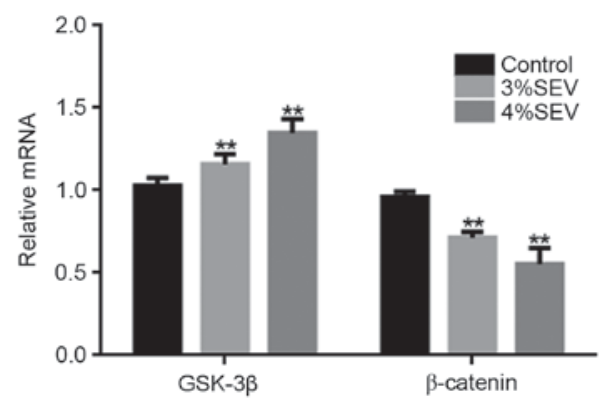

Figure 4. SEV inhibited the proliferation of neurons in juvenile rats potentially via Wnt signaling-associated pathways. The (A) protein and (B) mRNA expression levels of GSK- $3 \beta$ and $\beta$-catenin in neurons of neonatal rats. ${ }^{* *} \mathrm{P}<0.05$ vs. control. SEV, sevoflurane; GSK-3 $\beta$, glycogen synthase kinase- $3 \beta$. 
addition, Wnt signaling is critical for neuronal maturation, apoptosis, dopaminergic and hippocampal neurogenesis (29). Wnt signaling increases $\beta$-catenin levels by inhibiting the activity of GSK-3 $\beta$. GSK-3 $\beta$ is a serine/threonine kinase that controls various neuronal functions, including neurite outgrowth, synapse formation, neurotransmission and neurogenesis $(30,31)$. GSK-3 $\beta$ mediates these functions by phosphorylating a wide range of substrates involved in gene transcription, metabolism, apoptosis, cytoskeletal dynamics, signal transduction, lipid membrane dynamics and trafficking $(32,33)$. $\beta$-catenin is a central component of the Wnt/ $\beta$-catenin signaling pathway, which transmits information to the cytoplasm and translocates to the nucleus in order to activate target gene transcription (34). Previous studies have suggested that the $\mathrm{Wnt} / \beta$-catenin pathway stimulates the self-renewal of neural stem cells and their differentiation towards neuronal phenotype (35) by inhibiting gliogenesis (36). Downregulation of this pathway may lead to striatal synaptic degeneration, resulting in impaired motor behavior (37). The present study demonstrated that the protein and mRNA expression levels of GSK-3 $\beta$ and $\beta$-catenin in sevoflurane-exposed groups were significantly increased compared with the control group. Therefore, it is possible that sevoflurane may suppress the proliferation of nerve cells in the offspring of rats exposed during pregnancy, potentially via inhibition of the Wnt/ $\beta$-catenin signaling pathway.

In conclusion, the results of the present study indicate that sevoflurane may inhibit the proliferation of neural cells and promote their apoptosis in the offspring of rats exposed during pregnancy. In addition, sevoflurane may affect the learning and memory abilities of the rat offspring. Furthermore, the $\mathrm{Wnt} / \beta$-catenin pathway may be involved in the negative effects of sevoflurane exposure in rat offspring. Ultimately, the exposure of pregnant mice to sevoflurane anesthesia demonstrated a negative effect on the learning and memory abilities of their offspring; an effect that may be meditated by the Wnt/ $\beta$-catenin signaling pathway.

\section{References}

1. Heesen $\mathrm{M}$ and Klimek M: Nonobstetric anesthesia during pregnancy. Curr Opin Anaesthesiol 29: 297-303, 2016.

2. Owsiak JN and Bullough AS: Chronic myeloid leukemia in pregnancy: An absolute contraindication to neuraxial anesthesia? Int J Obstet Anesth 25: 85-88, 2016.

3. Miller TL, Park R and Sun LS: Report on the Fifth PANDA Symposium on 'Anesthesia and Neurodevelopment in Children'. J Neurosurg Anesthesiol 28: 350-355, 2016.

4. Stratmann G: Review article: Neurotoxicity of anesthetic drugs in the developing brain. Anesth Analg 113: 1170-1179, 2011.

5. Costi D, Cyna AM, Ahmed S, Stephens K, Strickland P, Ellwood J, Larsson JN, Chooi C, Burgoyne LL and Middleton P: Effects of sevoflurane versus other general anaesthesia on emergence agitation in children. Cochrane Database Syst Rev CD007084, 2014.

6. Lorsomradee S, Cromheecke S, Lorsomradee S and De Hert SG: Effects of sevoflurane on biomechanical markers of hepatic and renal dysfunction after coronary artery surgery. J Cardiothorac Vasc Anesth 20: 684-690, 2006.

7. Head BP and Patel P: Anesthetics and brain protection. Curr Opin Anaesthesiol 20: 395-399, 2007.

8. Istaphanous GK, Howard J, Nan X, Hughes EA, McCann JC, McAuliffe JJ, Danzer SC and Loepke AW: Comparison of the neuroapoptotic properties of equipotent anesthetic concentrations of desflurane, isoflurane, or sevoflurane in neonatal mice. Anesthesiology 114: 578-587, 2011.
9. Shen X, Dong Y, Xu Z, Wang H, Miao C, Soriano SG, Sun D, Baxter MG, Zhang Y and Xie Z: Selective anesthesia-induced neuroinflammation in developing mouse brain and cognitive impairment. Anesthesiology 118: 502-515, 2013.

10. Li Y, Zeng M, Chen W, Liu C, Wang F, Han X, Zuo Z and Peng S: Dexmedetomidine reduces isoflurane-induced neuroapoptosis partly by preserving PI3K/Akt pathway in the hippocampus of neonatal rats. PLoS One 9: e93639, 2014.

11. Joksimovic $M$ and Awatramani R: Wnt $/ \beta$-catenin signaling in midbrain dopaminergic neuron specification and neurogenesis. J Mol Cell Biol 6: 27-33, 2014.

12. Parish CL and Thompson LH: Modulating Wnt signaling to improve cell replacement therapy for Parkinson's disease. J Mol Cell Biol 6: 54-63, 2014.

13. Lange C, Mix E, Rateitschak K and Rolfs A: Wnt signal pathways and neural stem cell differentiation. Neurodegener Dis 3: 76-86, 2006.

14. Dai ZM, Sun S, Wang C, Huang H, Hu X, Zhang Z, Lu QR and Qiu M: Stage-specific regulation of oligodendrocyte development by Wnt//-catenin signaling. J Neurosci 34: 8467-8473, 2014.

15. L'Episcopo F, Tirolo C, Testa N, Caniglia S, Morale MC, Deleidi M, Serapide MF, Pluchino S and Marchetti B: Plasticity of subventricular zone neuroprogenitors in MPTP (1-methyl-4-phenyl-1,2,3,6-tetrahydropyridine) mouse model of Parkinson's disease involves cross talk between inflammatory and Wnt/ $\beta$-catenin signaling pathways: Functional consequences for neuroprotection and repair. J Neurosci 32: 2062-2085, 2012.

16. Liu $\mathrm{H}$ and Lou W: Functioning remobilization of the paralyzed vocal cord using the split-vagus nerve procedure in rats the split-vagus nerve procedure in rats. Lin Chung Er Bi Yan Hou Tou Jing Wai Ke Za Zhi 24: 273-275, 2010 (In Chinese).

17. Schoenfeld R, Schiffelholz T, Beyer C, Leplow B and Foreman N: Variations of the Morris water maze task to comparatively assess human and rodent place navigation. Neurobiol Learn Mem 139: 117-127, 2017.

18. Radahmadi M, Alaei H, Sharifi MR and Hosseini N: Effect of forced exercise and exercise withdrawal on memory, serum and hippocampal corticosterone levels in rats. Exp Brain Res 233: 2789-2799, 2015.

19. Livak KJ and Schmittgen TD: Analysis of relative gene expression data using real-time quantitative PCR and the 2(-Delta Delta C(T)) Method. Methods 25: 402-408, 2001.

20. Patel D and Chaudhary J: Increased expression of bHLH transcription factor E2A (TCF3) in prostate cancer promotes proliferation and confers resistance to doxorubicin induced apoptosis. Biochem Biophys Res Commun 422: 146-151, 2012.

21. Lerman J, Sikich N, Kleinman S and Yentis S: The pharmacology of sevoflurane in infants and children. Anesthesiology 80: 814-824, 1994.

22. Liang G, Ward C, Peng J, Zhao Y, Huang B and Wei H: Isoflurane causes greater neurodegeneration than an equivalent exposure of sevoflurane in the developing brain of neonatal mice. Anesthesiology 112: 1325-1334, 2010.

23. Shen X, Liu Y, Xu S, Zhao Q, Guo X, Shen R and Wang F: Early life exposure to sevoflurane impairs adulthood spatial memory in the rat. Neurotoxicology 39: 45-56, 2013.

24. Takaenoki Y, Satoh Y, Araki Y, Kodama M, Yonamine R, Yufune $S$ and Kazama T: Neonatal exposure to sevoflurane in mice causes deficits in maternal behavior later in adulthood. Anesthesiology 120: 403-415, 2014.

25. Kruger K, Stefansson IM, Collett K, Arnes JB, Aas T and Akslen LA: Microvessel proliferation by co-expression of endothelial nestin and $\mathrm{Ki}-67$ is associated with a basal-like phenotype and aggressive features in breast cancer. Breast 22: 282-288, 2013.

26. Hirabayashi Y, Itoh Y, Tabata H, Nakajima K, Akiyama T, Masuyama $\mathrm{N}$ and Gotoh Y: The Wnt/beta-catenin pathway directs neuronal differentiation of cortical neural precursor cells. Development 131: 2791-2801, 2004.

27. Rudloff $S$ and Kemler R: Differential requirements for $\beta$-catenin during mouse development. Development 139: 3711-3721, 2012.

28. Lupo G, Novorol C, Smith JR, Vallier L, Miranda E, Alexander M, Biagioni S, Pedersen RA and Harris WA: Multiple roles of Activin/Nodal, bone morphogenetic protein, fibroblast growth factor and Wnt/ $\beta$-catenin signalling in the anterior neural patterning of adherent human embryonic stem cell cultures. Open Biol 3: 120167, 2013.

29. Castelo-Branco G and Arenas E: Function of Wnts in dopaminergic neuron development. Neurodegener Dis 3: 5-11, 2006. 
30. Cole AR: GSK3 as a Sensor Determining Cell Fate in the Brain Front Mol Neurosci 5: 4, 2012.

31. Fuchs C, Trazzi S, Torricella R, Viggiano R, De Franceschi M, Amendola E, Gross C, Calzà L, Bartesaghi R and Ciani E: Loss of CDKL5 impairs survival and dendritic growth of newborn neurons by altering AKT/GSK-3 $\beta$ signaling. Neurobiol Dis 70: 53-68, 2014.

32. Kim WY, Wang X, Wu Y, Doble BW, Patel S, Woodgett JR and Snider WD: GSK-3 is a master regulator of neural progenitor homeostasis. Nat Neurosci 12: 1390-1397, 2009.

33. Morales-Garcia JA, Luna-Medina R, Alonso-Gil S, Sanz-Sancristobal M, Palomo V, Gil C, Santos A, Martinez A and Perez-Castillo A: Glycogen synthase kinase 3 inhibition promotes adult hippocampal neurogenesis in vitro and in vivo. ACS Chem Neurosci 3: 963-971, 2012.
34. Munji RN, Choe Y, Li G, Siegenthaler JA and Pleasure SJ: Wnt signaling regulates neuronal differentiation of cortical intermediate progenitors. J Neurosci 31: 1676-1687, 2011.

35. Kalani MY, Cheshier SH, Cord BJ, Bababeygy SR, Vogel H, Weissman IL, Palmer TD and Nusse R: Wnt-mediated self-renewal of neural stem/progenitor cells. Proc Natl Acad Sci USA 105: 16970-16975, 2008.

36. Kunke D, Bryja V, Mygland L, Arenas E and Krauss S: Inhibition of canonical Wnt signaling promotes gliogenesis in P0-NSCs. Biochem Biophys Res Commun 386: 628-633, 2009.

37. Galli S, Lopes DM, Ammari R, Kopra J, Millar SE, Gibb A and Salinas PC: Deficient Wnt signalling triggers striatal synaptic degeneration and impaired motor behaviour in adult mice. Nat Commun 5: 4992, 2014. 